\title{
Concomitant Pharmacotherapy of Psychotropic Medications in EU Children and Adolescents with Attention-Deficit/ Hyperactivity Disorder
}

\author{
Vanja Sikirica • Moshe Fridman • Amanda Bruno • \\ Paul Hodgkins $\cdot$ M. Haim Erder
}

Published online: 23 November 2013

(C) The Author(s) 2013. This article is published with open access at Springerlink.com

\begin{abstract}
Background With preliminary data suggesting an increasing trend in attention-deficit/hyperactivity disorder (ADHD) prevalence in Europe, the use of psychotropic medications in this population needs to be better understood, particularly among patients with ADHD and no comorbid psychiatric disorder.

Methods Medical charts of patients aged 6-17 years with one or more ADHD diagnosis between January 1, 2004 and June 30, 2007, and use of ADHD medication were abstracted by physicians from six European countries. Patients with a history of epilepsy or diagnosis of Tourette syndrome were excluded.

Results Among a convenience sample of 569 children/ adolescent patients (mean age, 12.1 years), 80 (14.1\%) patients used psychotropic concomitant medication (PCM) along with their current on-label ADHD medication. The number of pre-existing co-morbidities, high impairment due to the symptom of anger, and country (France; Italy; the Netherlands; and Spain vs. the reference country, Germany) were significantly associated with PCM use (UK was not significantly different vs. Germany). In particular, in France, Italy, the Netherlands, and Spain, PCM use was highest.
\end{abstract}

\footnotetext{
V. Sikirica $(\bowtie) \cdot$ P. Hodgkins $\cdot$ M. H. Erder Global Health Economics Outcomes Research and Epidemiology, Shire, 725 Chesterbrook Boulevard, Wayne, PA 19087, USA

e-mail: vsikirica@shire.com

M. Fridman

AMF Consulting, Inc., Los Angeles, CA, USA
}

A. Bruno

Xcenda, LLC, Palm Harbor, FL, USA
Conclusions These findings suggest that greater attention to the use of PCM, which are not indicated for the treatment of ADHD, may be warranted in children and adolescents receiving PCM. This highlights the need for further research to assess the impact of PCM use in ADHD patients and to consider alternative, individualized, indicated treatment strategies for patients with ADHD.

\section{Introduction}

\subsection{Attention-Deficit/Hyperactivity Disorder Treatment Options and Guidelines}

In children, adolescents, and adults, attention-deficit/ hyperactivity disorder (ADHD) is a heterogeneous behavioral disorder characterized by the presence of core symptoms of inattention, hyperactivity, and impulsivity [1]. While it is common for these core symptoms to present together, symptoms of ADHD can also overlap with symptoms of other related disorders and common coexisting conditions, such as learning disability, oppositional defiant disorder (ODD), conduct disorder, anxiety, depression, bipolar disorder, Tourette syndrome, substance abuse, or others [1, 2].

In Europe, study-reported prevalence rates of ADHD in individual countries, in the range of $2.8-7.3 \%$ (France $7.3 \%$; Germany $3.1 \%$; Italy $2.8 \%$; the Netherlands $5.0 \%$ ), have been increasing in recent years [3-5]. In the UK, data from the British Child and Adolescent Mental Health Survey of parents, teachers, and children indicated that $3.6 \%$ of boys and $0.85 \%$ of girls between the ages of 5 and 15 years have ADHD [6]. With a large degree of variation in clinical presentation and a high risk for cooccurring disorders [1,7], some European guidelines [e.g., 
National Institute for Clinical Healthcare and Excellence (NICE), Leitlinie der Arbeitsgemeinschaft ADHS der Kinder- und Jugendärzte $\mathrm{eV}$, Guidelines of the Italian Society of Neuropsichiatria dell'Infanzia and Adolescence (SINPIA), the British Association for Psychopharmacology] require a clinician with special training, such as a child psychiatrist, to make or confirm a diagnosis of ADHD [6]. Many studies have demonstrated the clinical efficacy and safety of pharmacotherapy as monotherapy, which is often prescribed for ADHD [8-11]. European guidelines recommend that optimal management of ADHD patients be based on a comprehensive treatment plan that includes some form of psychosocial intervention with or without medication $[1,12-15]$.

In patients with severe ADHD, pharmacologic treatment is an option, whereas for patients who are less severe, psychosocial interventions, such as behavioral therapy, should be tried first $[2,6]$. When pharmacologic treatment is indicated, the European guidelines unanimously recommend the use of stimulants in children, adolescents, and adults as a first-line pharmacologic therapy [12]. Atomoxetine, or other nonstimulant therapies, such as clonidine and guanfacine, are recognized as alternatives in most European guidelines [2, 6, 12,14] and are listed as first-line pharmacologic treatment options for: (1) adults with ADHD who began treatment in childhood; (2) when parent or patient preference is to not use a stimulant; (3) among patients who fail to respond or have a sub-optimal response to stimulants; or (4) when a patient has co-morbid substance abuse, tics, or anxiety [2, 12-14, 16]. Among school-age children, adolescents, and adults with severe ADHD [12, 15], several European guidelines recommend adopting a multimodal treatment plan $[13,15,17,18]$ that may include methylphenidate, atomoxetine, or dexamfetamine, depending on country-specific availability [6].

\subsection{Coexisting Conditions and Concomitant Drug Therapy}

Despite published guidelines on the use of pharmacotherapy and multimodal treatment plans for ADHD, few recommendations exist for children and adolescents who do not respond in part or fully to recommended therapies, and even less is known about the impact of adding on other pharmacotherapies for treating ADHD. While seeking treatment early for ADHD symptoms may improve ADHD-related outcomes in children and adolescents [16, 19], the symptoms of ADHD often overlap with co-existing developmental and psychiatric disorders [14, 20, 21], thus increasing the importance of making optimal treatment decisions for these ADHD patients.

Even though concomitant psychotropic medications are not indicated according to their product label for use in children and adolescents in the treatment of ADHD [22], European and US studies have reported their off-label use in this population [23]. A retrospective study of prescription medical records data in the Netherlands reported that antipsychotics $(6 \%)$ and melatonin $(4 \%)$ were the most commonly used therapeutics in the year before ADHD treatment initiation [4]. Another study conducted in the Netherlands reported that users of ADHD medication had used atypical antipsychotics at a rate of $5 \%$, while users of lithium, valproate, and lamotrigine had tried ADHD medication at a rate of $20-26 \%$ and even used these drugs concomitantly (15-21\%) [21]. A Danish study found that antidepressants and antipsychotics were used at rates of $4.9 \%$ and $7.1 \%$, respectively, among patients under the age of 18 years with ADHD who also received medication within the Anatomical Therapeutic Chemical classification of the nervous system [24]. Further, a study among Italian children and adolescents receiving ADHD medication reported a $22 \%$ rate of concomitant psychotropic medication use based on registry data from Northern Italy [25].

With two recent studies suggesting an increasing trend in ADHD prevalence in Europe [6, 25] and little documentation of concomitant use of psychotropic drugs in this population, the use of psychotropic medications needs to be better understood, particularly among those patients where their use is for ADHD - that is, patients with ADHD and no co-morbid psychiatric disorder. As such, the purpose of this study was to estimate the rates of psychotropic concomitant medication (PCM) use in six European countries and to identify patient characteristics associated with PCM use among children and adolescents receiving a product label-indicated ADHD treatment.

\section{Methods}

\subsection{Study Data and Selection Criteria}

This retrospective cohort study is based on a review and data abstraction of patient medical records by their treating physicians in six Western European countries: the UK, France, Germany, Italy, the Netherlands, and Spain. A convenience sample of pediatricians, neuropediatricians, child and/or adolescent psychiatrists, and pediatric neurologists who treated patients with ADHD was identified from physician directories maintained by local country medical associations and physician telephone directories. Physicians included in the database were recruited by telephone or email and directed to an Internet-based questionnaire to potentially participate in the study. Physicians with between 3 and 30 years of experience were eligible for inclusion if they managed a minimum of five patients per month with ADHD between the ages of 6 and 
17 years and were primarily responsible for making ADHD-related treatment decisions for the patient.

Institutional Review Board study protocol review and exemption was obtained prior to study data collection. All data were entered by the physician via an online questionnaire translated into the language of the country. Physicians were asked to complete an ADHD patient chart review for up to five of their most recent patients who met the patient study age criterion, had a documented diagnosis of ADHD between January 1, 2004 and June 30, 2007, and had at least 2 consecutive years of follow-up post-diagnosis (e.g., medical record information available). Patients were also required to have received either pharmacologic treatment or behavioral therapy following the ADHD diagnosis. Eligible patient charts could have a diagnosis of ADHD only, or ADHD combined with the presence of other behavioral symptoms (e.g., anger, irritability), related behavioral disorders (e.g., ODD), or psychiatric co-morbidities (e.g., autism, anxiety). Symptom impairment scale responses were evaluated in the range of 1 being the "lowest impairment" to 10 being the "highest impairment." Patient charts were excluded if there was evidence of enrollment in a randomized clinical trial during the time of the data abstraction. For purposes of this analysis, additional criteria were applied to increase the likelihood that PCM was used for ADHD. Patients with pre-existing epilepsy or Tourette syndrome were excluded as these are concomitant conditions that may warrant the use of psychotropic medications such as neuroleptic or antiepileptic drugs, which could have been used for both ADHD and these concomitant conditions. In addition, patients receiving behavioral therapy alone were excluded.

\subsection{Psychotropic Concomitant Medication (PCM) Use}

Patients receiving both a product label-indicated ADHD medication (with or without behavioral therapy) and any psychotropic medication (with no product label claim for ADHD) during current ADHD treatment-i.e., the treatment the patient was receiving at the time of chart review-were classified as PCM users. Patients receiving product label-indicated ADHD medication (with or without behavioral therapy) and no PCM during current ADHD treatment were classified as ADHD medication-only patients. ADHD medication-only patients could have used a combination of ADHD medications that were approved by the European Medicines Agency that also had a product label claim for the treatment of ADHD as long as there was no other psychotropic medication used. The psychotropic medications included medications that may have been used but that did not contain a product label claim for ADHD: selective serotonin reuptake inhibitors (SSRIs), serotonin norepinephrine reuptake inhibitors (SNRIs), tricyclic antidepressants (TCAs), monoamine oxidase (MAO) inhibitors, typical antipsychotics, atypical antipsychotics, benzodiazepine/anxiolytics, $\alpha-2$ agonists clonidine and guanfacine, and antiepileptic drugs (without epilepsy diagnosis).

\subsection{Statistical Analysis of PCM Use}

Pooled analyses across countries were performed to increase sample size. Analyses were also conducted within country, and use was described by specific type of medication class. The significance of the relationships between baseline patient characteristics and PCM use was tested using the Fisher's exact test or $t$ tests for dichotomous and continuous variables, respectively. All statistical tests were two-sided, and $P$ values $\leq 0.05$ were considered statistically significant. Data were summarized using descriptive statistics for continuous variables and frequency and percentage for categorical variables.

\subsection{Patient Characteristics Associated with PCM Use}

To identify patient characteristics associated with PCM use, analyses focused on comparisons of patients who received PCM with their current ADHD treatment with those who did not. A multiple logistic regression model for current PCM use was fitted to assess the simultaneous effect of baseline patient and treatment characteristics from the list of covariates that tested significant in individual bivariate tests for the outcome. This was done to limit multi-collinearity and over-fitting of the model given that the number of observations (e.g., sample size) may not have been sufficiently large to allow for each individual variable to be entered into the model. Selection of covariates was performed using the stepwise variable selection procedure with stay and remove at significance levels of $P<0.05$. The selection results were verified using the backwards elimination method. Interactions and secondorder terms (continuous covariates only) for covariates testing significantly over and above the main effects were retained. $C$-statistics were reported as a measure of the model's accuracy of prediction [26].

\subsection{Sensitivity Analyses}

To test the robustness of the base case rate of PCM use, several subsets of patients were also examined. The first analysis excluded pre-existing schizophrenia or obsessivecompulsive disorder (OCD), in addition to the already excluded epilepsy and Tourette syndrome patients. The second analysis excluded patients with evidence of preexisting schizophrenia, OCD, epilepsy, Tourette syndrome, autism, alcohol abuse, or substance abuse. To test the most 
extreme possibilities, all patients with any co-morbidity, except ODD, were removed and a rate calculated. The effect of adding all patients with behavioral therapy only (and not on ADHD pharmacotherapy) to the base case denominator on the rate of PCM use was also examined. Country-specific rates of PCM use for these patients with behavioral therapy alone were examined relative to the original patient sample.

One last sensitivity analysis was conducted to assess the impact of age on PCM use. Specifically, because children (aged 6-12 years) and adolescents (aged 13-17 years) are often quite different in clinical presentation, interaction terms by age group were tested in the multivariate regression models on PCM use.

\section{Results}

\subsection{Patient Characteristics Associated with PCM Use}

Of the 730 total charts of patients treated for ADHD in the dataset, 42 patients with epilepsy $(n=3)$ or Tourette syndrome $(n=39)$ were excluded; and of the remaining 689 charts, an additional 120 patients were excluded for not using any ADHD medication with a product label claim at the time of chart review (e.g., behavioral therapy only). Therefore, a total of 569 patient charts from 283 physicians were identified as meeting selection criteria from all six countries. Overall, 80 (14.1\%) patients were PCM users, and the remaining 489 only used ADHD-labeled medication(s); $22.7 \%$ of the 569 patients were female, and the mean age was 12.1 years. Differences in gender and age across countries were not statistically significant (data not shown). Atypical antipsychotics were the most commonly used PCM (4.0 \% overall, $28.8 \%$ of PCM users); followed by anxiolytics (3.9\% overall, $27.5 \%$ of PCM users); melatonin (2.1\% overall, $15.0 \%$ of PCM users); SSRIs (1.8\% overall, $12.5 \%$ of PCM users); typical antipsychotics (1.4\% overall, $10.0 \%$ of PCM users); clonidine ( $0.9 \%$ overall, $6.3 \%$ of PCM users), and SNRIs, TCAs, MAO inhibitors, antiepileptic drugs, and a general "other" category (each $0.4 \%$ overall or $2.5 \%$ of PCM users) (Fig. 1). Note that the percentages overall and among PCM users are not mutually exclusive, as the same patient could have been counted in more than one PCM category. The rate of PCM use differed across countries $(P<0.0001)$, with the lowest rate occurring in Germany at $4.1 \%$ $(P<0.0001)$ and the highest rate in Italy at $32.7 \%$ $(P<0.0001)$. The high rate in Italy was primarily driven by the high rate of anxiolytic use $(23.1 \%)$.

At baseline, PCM users had significantly higher rates of anxiety, depression, bipolar disorder, aggression, OCD, insomnia, ODD, and learning disability (Fig. 2). PCM
口SSRIs Anxiolytics $\square$ Antipsychotic $\square$ Other $\square$ Melatonin

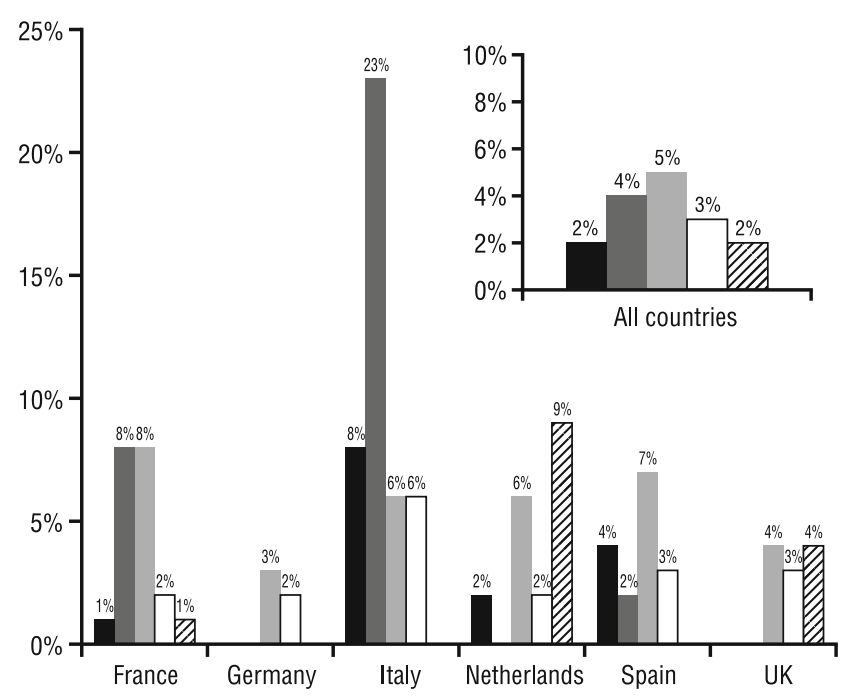

Fig. 1 PCM use by country. Percentages represent proportion of groups for which data were available. Other includes clonidine (clonidine use: UK, $3.4 \%$; the Netherlands, $1.6 \%$; all other countries, $0 \%$ ), SNRIs, TCAs, MAO inhibitors, antiepileptic drugs, and a general "other" category. Categories were not mutually exclusive, thus the same patient could be counted in multiple categories. Total percentages of PCM use by country were the following: Italy $32.7 \%$, France $19.0 \%$, the Netherlands $15.6 \%$, Spain $14.2 \%$, UK $11.0 \%$, and Germany $4.1 \%$. PCM psychotropic concomitant medication, SSRI selective serotonin reuptake inhibitor, SNRI serotonin norepinephrine reuptake inhibitor, TCI tricyclic antidepressant, $M A O$ monoamine oxidase

users were also significantly older (59\% aged 13-17 years vs. $41 \%$ aged $6-12$ years, $P=0.005)$ and had a higher number of pre-existing co-morbidities (mean 3.7 vs. 2.4, $P<0.0001)$ compared with the ADHD medication-only group (Table 1). In addition, the rate of ADHD symptoms at diagnosis differed between groups: PCM users had higher rates of anger, irritability, and inappropriate behavior, and also exhibited higher overall mean impairment level (mean 7.2 vs. $6.3, P<0.0001$ ) than the group with ADHD medication only. PCM users also had a higher physician-reported rate of concurrent behavioral therapy (60 vs. $38 \%, P=0.0004$ ) and lower levels of patient engagement ( 6.0 vs. $6.6, P=0.010$ ). Race; education; inschool status; employment; and ADHD among siblings, parents, or other family members were not significantly different between groups. Other factors that were similar between groups included evidence of impairment at work, school, or social settings; number of years since diagnosis; number of treatment lines per follow-up year; and level of family involvement in the patient's ADHD condition and treatment.

After controlling for baseline covariates in the multiple logistic regression model $(C$-statistic $=0.76)$, several variables remained significant predictors of PCM use, 
Fig. 2 Co-morbidities by medication group. PCM psychotropic concomitant medication, $A D H D$ attentiondeficit/hyperactivity disorder, $O D D$ oppositional defiant disorder
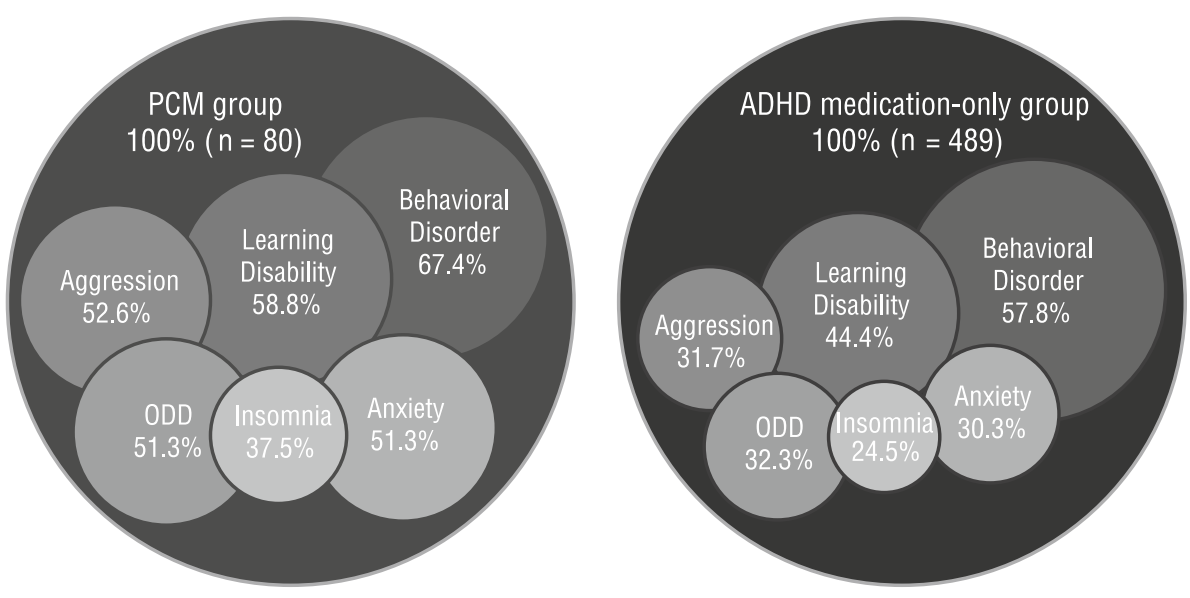

including the number of pre-existing co-morbidities [odds ratio; OR (95\% confidence interval; CI) $=1.16(1.01$, 1.33), $P=0.03$ ], high impairment due to symptom of anger [OR $(95 \% \mathrm{CI})=1.79(1.29,2.47)$ per 1 standard deviation increase, $P=0.0005$ ], and country [France: OR $(95 \% \mathrm{CI})=3.37(1.16,9.75), P=0.03$; Italy: OR $(95 \%$ $\mathrm{CI})=5.11(1.65,15.79), P=0.005$; the Netherlands: OR $(95 \% \mathrm{CI})=3.74(1.18,11.78), P=0.025$; and Spain: OR $(95 \% \mathrm{CI})=3.73(1.18,11.78), P=0.02$ vs. the reference country, Germany]. Age group was significant (overall $P=0.035$ ) with patients aged 13-17 years having a 1.72fold (95\% CI; 0.84, 3.50) higher odds of PCM use compared with patients aged 6-9 years. Figure 3 shows the estimated probability curves for PCM use by number of pre-existing co-morbidities predicted by the multiple logistic regression estimated equation using patient charts in Spain as an example; first quartile (scored as 3 out of 10) and third quartile (scored as 8 out of 10) anger impairment scores were used as representative fixed values for all sample-estimated probability curves and modeled in combination with age group. Accordingly, a patient from Spain aged 13-17 years with three co-morbidities and low anger impairment (25th percentile score of 3 out of 10) would have a $14 \%$ estimated probability of receiving PCM versus $32 \%$ for an identical patient with higher anger impairment (75th percentile score of 8 out of 10 ).

\subsection{Sensitivity Analysis Results}

In the base case analysis, our sample of children and adolescents without epilepsy or Tourette syndrome $(n=569)$, a $14.1 \%$ (95\% CI; $11.2,17.0 \%)$ rate of PCM use was observed. In the first subset analysis, 541 patients remained after patients with pre-existing schizophrenia or OCD $(n=28)$ were excluded. In the second subset analysis, 512 patients remained after patients with evidence of pre-existing schizophrenia, OCD, epilepsy, Tourette syndrome, autism, alcohol abuse, or substance abuse were excluded $(n=57)$. The rate of PCM use among both of these subsets was $13.3 \%$ (95\% CIs; 10.4, $16.2 \%$ for both subsets).

To test the most extreme possibility, when all patients with any co-morbidity except ODD were removed, the PCM use rate was $7.9 \%$ (10 patients of 126, $95 \%$ CI; 3.2, $12.7 \%$ ). Additionally, once patients with behavioral therapy only (not on ADHD pharmacotherapy; $n=120$ ) were added back to the original base case analysis $(n=689)$, the rate of PCM use was $11.6 \%$ (80 patients of $689,95 \% \mathrm{CI}$; $9.2,14.0 \%$ ). Comparison of country-specific rates of PCM use including patients with behavioral therapy only in the denominator (relative to the overall rate of $11.6 \%$ across countries) was in the range of $3.4 \%$ (Germany; $P<0.0001)$ to $15.9 \%$ (Italy; not significant). These were similar to rates of PCM use in the original patient subgroup (excluding behavioral therapy).

Last, because children (aged 6-12 years) and adolescents (aged 13-17 years) were observed to be quite different in terms of country and other baseline factors; multivariate regression models and interaction terms by age group were tested on PCM use and none of these terms were statistically significant, thus indicating that the differences in country or co-morbidity by age group were not significant factors related to PCM use.

\section{Discussion}

Results from this study of six European countries indicated that $14.1 \%$ of children and adolescents diagnosed with and receiving medication for ADHD with no behavioral treatment were treated concomitantly with psychotropic therapies, even though the psychiatric therapies were not product label indicated for ADHD treatment across Europe. The PCM rate of $14.1 \%$ was observed in the sample of children and adolescents without epilepsy or Tourette syndrome and dropped less than a full percentage point 
Table 1 Baseline characteristics by current PCM use

\begin{tabular}{|c|c|c|c|}
\hline Baseline characteristics & $\begin{array}{l}\text { PCM use } \\
n=80\end{array}$ & $\begin{array}{l}\text { ADHD medication only } \\
n=489\end{array}$ & $P$ value \\
\hline Age group $[n(\%)]$ & & & 0.0047 \\
\hline $6-9$ years & $13(16.3)$ & $82(16.8)$ & \\
\hline $10-12$ years & $20(25.0)$ & 209 (42.7) & \\
\hline 13-17 years & $47(58.8)$ & $198(40.5)$ & \\
\hline Gender $[n(\%)]$ & & & 0.7751 \\
\hline Male & $61(76.3)$ & $379(77.5)$ & \\
\hline Female & $19(23.8)$ & $110(22.5)$ & \\
\hline Country $[n(\%)]$ & & & $<0.0001$ \\
\hline France & $19(23.8)$ & $81(16.6)$ & \\
\hline Italy & $17(21.3)$ & $35(7.2)$ & \\
\hline Spain & $16(20.0)$ & $97(19.8)$ & \\
\hline UK & $13(16.3)$ & $106(21.7)$ & \\
\hline The Netherlands & $10(12.5)$ & $54(11.0)$ & \\
\hline Germany & $5(6.3)$ & $116(23.7)$ & \\
\hline \multicolumn{4}{|c|}{ Predominant symptoms/behaviors at diagnosis $[n(\%)]$} \\
\hline Inattention & $64(80.0)$ & $394(80.6)$ & 0.8798 \\
\hline Hyperactivity & $58(72.5)$ & $339(69.3)$ & 0.6020 \\
\hline Impulsivity & $59(73.8)$ & $326(66.7)$ & 0.2463 \\
\hline Anger & $37(46.3)$ & $168(34.4)$ & 0.0447 \\
\hline Irritability & $48(60.0)$ & $196(40.1)$ & 0.0010 \\
\hline Active defiance of reasonable requests & $36(45.0)$ & $197(40.3)$ & 0.4626 \\
\hline Tendency to blame other people & $20(25.0)$ & $89(18.2)$ & 0.1677 \\
\hline Challenges with school/work performance & $60(75.0)$ & $363(74.2)$ & 1.0000 \\
\hline Social problems when interacting & $50(62.5)$ & $272(55.6)$ & 0.2747 \\
\hline Difficulty making the right choices & $23(28.8)$ & $113(23.1)$ & 0.3218 \\
\hline Inappropriate behavior & $48(60.0)$ & $215(44.0)$ & 0.0107 \\
\hline Other & $3(3.8)$ & $19(3.9)$ & 1.0000 \\
\hline Sleeping troubles & $0(0.0)$ & $4(0.8)$ & 1.0000 \\
\hline Any core symptoms & $77(96.3)$ & $476(97.3)$ & 0.4812 \\
\hline Any behavioral symptoms & $78(97.5)$ & $463(94.7)$ & 0.4056 \\
\hline Currently on behavioral therapy $[n(\%)]$ & & & 0.0004 \\
\hline Yes & $48(60.0)$ & $188(38.4)$ & \\
\hline No & $32(40.0)$ & $301(61.6)$ & \\
\hline \multicolumn{4}{|c|}{ ADHD impairment level $^{\mathrm{a}}$ (scale 1-10), mean (SD) } \\
\hline Inattention & $7.91(1.77)$ & $7.79(1.70)$ & 0.5374 \\
\hline Hyperactivity & $7.63(2.13)$ & $7.13(2.20)$ & 0.0597 \\
\hline Impulsivity & $7.55(2.26)$ & $6.79(2.36)$ & 0.0074 \\
\hline Anger & $7.00(2.44)$ & $5.27(2.54)$ & $<0.0001$ \\
\hline Irritability & $6.85(2.61)$ & $5.69(2.42)$ & $<0.0001$ \\
\hline Defiance & $7.06(2.20)$ & $5.88(2.45)$ & $<0.0001$ \\
\hline Blame others & $5.68(2.48)$ & $4.64(2.43)$ & 0.0004 \\
\hline School/work performance & $7.86(1.93)$ & $7.73(1.71)$ & 0.5418 \\
\hline Social interactions & $7.60(2.10)$ & $6.77(2.21)$ & 0.0017 \\
\hline Making right choices & $6.41(2.12)$ & $5.45(2.16)$ & 0.0002 \\
\hline Inappropriate behavior & $7.24(2.17)$ & $6.28(2.23)$ & 0.0004 \\
\hline Other symptoms & 7.67 (2.08) & $8.16(1.95)$ & 0.6916 \\
\hline
\end{tabular}


Table 1 continued

\begin{tabular}{|c|c|c|c|}
\hline Baseline characteristics & $\begin{array}{l}\text { PCM use } \\
n=80\end{array}$ & $\begin{array}{l}\text { ADHD medication only } \\
n=489\end{array}$ & $P$ value \\
\hline \multicolumn{4}{|l|}{ Mean ADHD symptoms level ${ }^{\mathrm{a}}$ (scale $\left.1-10\right)$, mean (SD) } \\
\hline ADHD core symptoms ${ }^{\mathrm{b}}$ & $7.70(1.59)$ & $7.23(1.54)$ & 0.0138 \\
\hline Behavior symptoms $^{c}$ & $6.96(1.57)$ & $5.96(1.61)$ & $<0.0001$ \\
\hline Other symptoms & $7.67(2.08)$ & $8.16(1.95)$ & 0.6916 \\
\hline All symptoms ${ }^{\mathrm{d}}$ & $7.16(1.47)$ & $6.32(1.43)$ & $<0.0001$ \\
\hline \multicolumn{4}{|l|}{ Other baseline characteristics } \\
\hline Number of pre-existing co-morbidities: mean (SD) & $3.69(2.16)$ & $2.39(1.94)$ & $<0.0001$ \\
\hline Patient engaged $^{\mathrm{a}}$ (scale $1-10$ ) mean (SD) & $6.00(2.28)$ & $6.61(1.95)$ & 0.0114 \\
\hline \multicolumn{4}{|c|}{$P C M$ psychotropic concomitant medication, $A D H D$ attention-deficit/hyperactivity disorder, $S D$ standard deviation } \\
\hline \multicolumn{4}{|c|}{${ }^{\text {a }}$ Scale from $1=$ lowest $/$ none to $10=$ highest } \\
\hline \multicolumn{4}{|c|}{ b Calculated as the mean impairment for hyperactivity, inattention, and impulsivity } \\
\hline \multicolumn{4}{|c|}{$\begin{array}{l}\text { c Calculated as the mean impairment for anger, irritability, active defiance, tendency to blame others, challenges with school/work performance, } \\
\text { social problems when interacting with family/teachers and peers/colleagues, or difficulty making right choices }\end{array}$} \\
\hline
\end{tabular}

$(13.3 \%)$, when examining sensitivity analyses with subsets of the children and adolescents who also had no reported evidence in their medical records of other pre-existing conditions, including schizophrenia, OCD, autism, alcohol abuse, or drug abuse. Furthermore, among all patient groups studied, the rate of PCM use was relatively stable and used to treat their ADHD, as reported by their treating physicians. By comparison, the administration rate of psychotropic medications, specifically second-generation antipsychotics, to children with ADHD as their only diagnosis was reported as $14 \%$ in a US study of Medicaidenrolled children [23]. Although this study did not provide details of the use of multiple medications, patients taking co-medications were included in the analyses. A slightly higher rate of PCM use by patients with ADHD and no psychiatric co-morbidities (18\%) was reported by a nationwide physician survey conducted in the Netherlands [27].

This study also found significant variation in PCM use across countries. Such a result is difficult to interpret and may relate to physician training and practice setting, national standards and insurance systems, treatment priorities, variability in other available resources such as family and community support or supportive educational settings, cultural norms, or differences in approved medications. For example, Italy had the highest rate of PCM observed during this time period and did not have any long-acting stimulants approved for use, which may indicate the use of other medications to fill a potential gap in treatment therapy. Across all countries, important baseline differences were noted among patients receiving PCM relative to those who had ADHD monotherapy, suggesting differences in demographic and clinical characteristics between segments of the ADHD population. During the study observation period, PCM patients had more co-morbidities, greater occurrence of certain predominant symptoms, more use of behavioral therapy, greater patient engagement, and greater symptom impairment. After controlling for these baseline differences, patients with more pre-existing psychiatric comorbidities or those who had a high level of impairment due to the symptom of anger were still more likely to receive PCM alongside their ADHD treatment.

To our knowledge, this is the first study to provide a detailed description of children and adolescents with ADHD receiving ADHD-indicated treatment and concomitant psychiatric medications across multiple Western European countries. Other studies have examined the rate of PCM in children and adolescents with ADHD but typically have been limited to a single region and have not reported whether the patients had concomitant diagnosis of psychiatric disorders [25]. The most common form of PCM recorded in our study was antipsychotics $(5.4 \%)$. Atypical antipsychotics have been studied as off-label treatment for ADHD [22] but are not recognized by current practice guidelines in Europe [2, 12, 14]. European guidelines do not recommend the use of any psychotropic medications for ADHD, as these therapies do not have an indication for ADHD in children and adolescents. Rather, most European guidelines recommend the use of stimulant therapy as firstline pharmacologic treatment among school-age children as part of a multimodal treatment plan, and non-stimulant therapy in certain circumstances (e.g., when patients have a suboptimal response or intolerable adverse effects with stimulants $[2,13,16])$.

A majority of ADHD patients will be treated with stimulants, which are an effective first-line treatment 


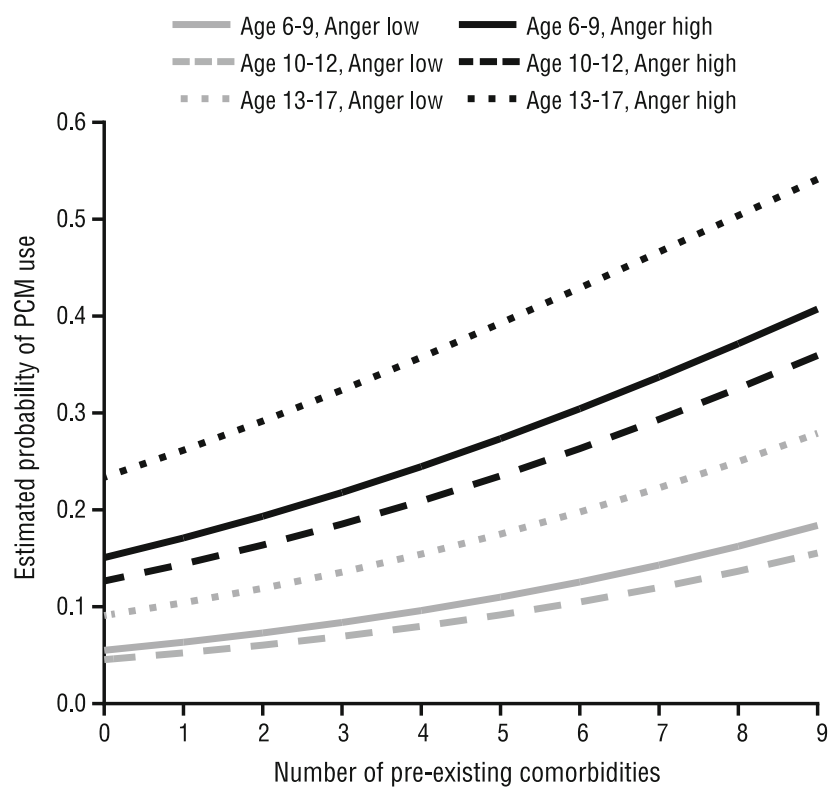

Fig. 3 Estimated probability of PCM use in patients from Spain by number of pre-existing co-morbidities, age group, and anger impairment level (logistic regression modeling). PCM psychotropic concomitant medication

option of which about $70 \%$ of patients will respond adequately $[28,29]$. However, approximately $30 \%$ of patients do not respond adequately to stimulant therapy and may require additional interventions, either pharmacologic or behavioral. As such, presently the use of PCM may fill some of this void; hence the outcomes of PCM use need to be better understood. Greater consideration should be given to developing individual treatment strategies that allow for different dosages and switching among different approved medications for ADHD, in contrast to the current practice of PCM use in ADHD with medications that do not have a product label indication for ADHD [2]. Such strategies would also allow the consideration of the complexities involved in managing ADHD, relying more extensively on clinical impression and partnerships with caretakers [30]. Consequently, further prospective studies are needed to better understand the use patterns of PCM in ADHD and the true impact of PCM in ADHD patients, caretakers, and their physicians.

The main strength of this study was the geographically wide pan-European population of children and adolescents with ADHD that represented six European countries and enabled a sufficient sample size to describe the rates and demographics from this convenience sample. The use of physician questionnaires, based on their own abstraction of their patient's medical record data, could have resulted in PCM use estimates that reflect real-world treatment patterns. In addition, the study design allowed for the collection of data not often collected in clinical trials or available in administrative claims databases.
This study contained certain limitations that must be considered alongside the results. There was no process verifying that the understanding of questions was uniform across clinicians, and thus different interpretations of responses for some variables may have occurred. In addition, physician responses on treatment outcome and other covariates may appear to be related, whereas if we had collected these data from various independent data sources, it is possible that correlations observed in this study would have been attenuated.

Physicians were asked if their patients received any of the following drugs for the treatment of ADHD. Physician responses were not confirmed by independent review of their medical records and their response may have depended on their individual interpretation of the question, which could result in the reporting of a PCM drug use for ADHD, when in effect it was used for another reason. This could possibly explain the observed correlation between baseline co-morbidities and increased use of PCM. Prospective studies are needed to further clarify this point. Another limitation of this study was the possibility of selection bias in the convenience sampling method used to select physicians and study groups at baseline. For instance, PCM proportions were different across countries, and PCM patients seemed to be more severe at baseline and to be diagnosed with more co-morbid illnesses. We descriptively compared the ADHD medication only group to the PCM users group as a normative control group. Within the analysis of patient characteristics associated with PCM use, we controlled for observed variables. However, neither analysis can control for unobserved differences and therefore the results of the analysis should be interpreted with care until further prospective confirmation of the study results are obtained.

Last, although ADHD was the only confirmed diagnosis common to all patients, it is possible that PCM may have been prescribed for the treatment of psychiatric co-morbidities (and not ADHD) for some patients. The sensitivity analysis for the subgroup of patients who had ADHD only reported in their medical records (with the exception of ODD) was conducted with this concern in mind. Yet, even in this subpopulation, there were $7.9 \%$ of patients prescribed PCM. To accurately assess the rate of patients prescribed PCM for ADHD only, a prospective study would have to be conducted; our data indicate that it occurs at some frequency.

\section{Conclusion}

This study found that $14.1 \%$ of children and adolescents in six Western European nations who received PCM for ADHD treatment received concomitant psychotropic 
medications that were not product indicated for ADHD. These rate results were generally robust in various sensitivity analyses. Patient-level factors associated with PCM use included the number of pre-existing co-morbidities and high impairment due to the symptom of anger.

Greater attention should be paid to the use of PCM, which are not indicated for the treatment of ADHD in children and adolescents. This may be particularly needed in France, Italy, the Netherlands, and Spain where PCM use was highest. Additional prospective research should be conducted to understand the implications of PCM use in patients with ADHD and to ensure that patients with ADHD are receiving optimal treatment and support.

Acknowledgments Funding for this research was provided by Shire Development LLC to Xcenda and AMF Consulting. Shire is a manufacturer of products that are used for the treatment of ADHD. VS, $\mathrm{PH}$, and MHE are employees of Shire and are stock/option owners of Shire. AB was an employee of Xcenda at the time of this study. MF is an independent statistical consultant with AMF Consulting. Melissa Brunckhorst, from MedErgy, provided editorial assistance in formatting, proofreading, and copy editing. This support was funded by Shire. Gina D'Angelo, PharmD, from Shire also reviewed and edited the manuscript for scientific accuracy. Although the sponsor was involved in the design, collection, analysis, interpretation, and fact checking of information, the content of this manuscript, the ultimate interpretation, and the decision to submit it for publication in Drugs in $R \& D$ were made by all the authors independently.

Conflict of interest VS, PH, and MHE are employees of Shire and hold stock/options in Shire. MF is an independent statistical consultant with AMF Consulting, which received funding from Shire Development LLC for this study. AB was an employee of Xcenda during the time of this study, which received funding from Shire Development LLC for this study.

Open Access This article is distributed under the terms of the Creative Commons Attribution Noncommercial License which permits any noncommercial use, distribution, and reproduction in any medium, provided the original author(s) and the source are credited.

\section{References}

1. National Institute of Mental Health. Attention deficit hyperactivity disorder (ADHD). 08-3572 ed. US Department of Health and Human Services; 2008. http://www.nimh.nih.gov/health/ publications/attention-deficit-hyperactivity-disorder/adhd_booklet_ c1508.pdf.

2. National Institute for Health and Clinical Excellence. Attention deficit hyperactivity disorder: the diagnosis and management of ADHD in children, young people and adults. NICE clinical guideline 72. 2008. p. 1-56. http://www.nice.org.uk/nicemedia/ pdf/cg72niceguidelinev3.pdf

3. Brod M, Pohlman B, Lasser R, Hodgkins P. Comparison of the burden of illness for adults with ADHD across seven countries: a qualitative study. Health Qual Life Outcomes. 2012;10(47).

4. Hodgkins P, Sasane R, Meijer WM. Pharmacologic treatment of attention-deficit/hyperactivity disorder in children: incidence, prevalence, and treatment patterns in the Netherlands. Clin Ther. 2011;33(2):188-203.
5. Polanczyk G, de Lima MS, Horta BL, Biederman J, Rohde LA. The worldwide prevalence of ADHD: a systematic review and metaregression analysis. Am J Psychiatry. 2007;164(6): $942-8$.

6. Atkinson M, Hollis C. NICE guideline: attention deficit hyperactivity disorder. Arch Dis Child Educ Pract Ed. 2010; 95(1):24-7.

7. The MTA Cooperative Group. Multimodal Treatment Study of Children with ADHD: a 14-month randomized clinical trial of treatment strategies for attention-deficit/hyperactivity disorder. Arch Gen Psychiatry. 1999;56(12):1073-86.

8. Barbaresi WJ, Katusic SK, Colligan RC, Weaver AL, Jacobsen SJ. Long-term school outcomes for children with attention-deficit/hyperactivity disorder: a population-based perspective. J Dev Behav Pediatr. 2007;28(4):265-73.

9. Biederman J, Melmed RD, Patel A, et al. A randomized, doubleblind, placebo-controlled study of guanfacine extended release in children and adolescents with attention-deficit/hyperactivity disorder. Pediatrics. 2008;121(1):e73-84.

10. Jain R, Segal S, Kollins SH, Khayrallah M. Clonidine extendedrelease tablets for pediatric patients with attention-deficit/hyperactivity disorder. J Am Acad Child Adolesc Psychiatry. 2011; 50(2):171-9.

11. Sallee FR, Lyne A, Wigal T, McGough JJ. Long-term safety and efficacy of guanfacine extended release in children and adolescents with attention-deficit/hyperactivity disorder. J Child Adolesc Psychopharmacol. 2009;19(3):215-26.

12. Seixas M, Weiss M, Muller U. Systematic review of national and international guidelines on attention-deficit hyperactivity disorder. J Psychopharmacol. 2012;26(6):753-65.

13. Banaschewski T, Coghill D, Santosh P, et al. Long-acting medications for the hyperkinetic disorders: a systematic review and European treatment guideline. Eur Child Adolesc Psychiatry. 2006;15(8):476-95.

14. Nutt DJ, Fone K, Asherson P, et al. Evidence-based guidelines for management of attention-deficit/hyperactivity disorder in adolescents in transition to adult services and in adults: recommendations from the British Association for Psychopharmacology. J Psychopharmacol. 2007;21(1):10-41.

15. Taylor E, Dopfner M, Sergeant J, et al. European clinical guidelines for hyperkinetic disorder: first upgrade. Eur Child Adolesc Psychiatry. 2004;13(Suppl 1):I7-30.

16. Scottish Intercollegiate Guidelines Network. Management of attention deficit and hyperkinetic disorders in children and young people: a national clinical guideline. 2009. http://www.sign.ac. uk/pdf/sign112.pdf.

17. Remschmidt H. Global consensus on ADHD/HKD. Eur Child Adolesc Psychiatry. 2005;14(3):127-37.

18. Kutcher S, Aman M, Brooks SJ, et al. International consensus statement on attention-deficit/hyperactivity disorder (ADHD) and disruptive behaviour disorders (DBDs): clinical implications and treatment practice suggestions. Eur Neuropsychopharmacol. 2004;14(1):11-28.

19. Molina BS, Hinshaw SP, Swanson JM, et al. The MTA at 8 years: prospective follow-up of children treated for combined-type ADHD in a multisite study. J Am Acad Child Adolesc Psychiatry. 2009;48(5):484-500.

20. Spencer TJ, Biederman J, Mick E. Attention-deficit/hyperactivity disorder: diagnosis, lifespan, comorbidities, and neurobiology. J Pediatr Psychol. 2007;32(6):631-42.

21. Bramness JG, Groholt B, Engeland A, Furu K. The use of lithium, valproate or lamotrigine for psychiatric conditions in children and adolescents in Norway 2004-2. J Affect Disord. 2009; 117(3):208-11.

22. Maglione M, Maher A, Hu J, et al. Off-label use of atypical antipsychotics: an update. Comp Eff Rev. 2011; 43. 
23. Matone M, Localio R, Huang YS, dosReis S, Feudtner C, Rubin D. The relationship between mental health diagnosis and treatment with second-generation antipsychotics over time: a national study of U.S. Medicaid-enrolled children. Health Serv Res. 2012;47(5):1836-60.

24. Pottegard A, Bjerregaard BK, Glintborg D, Kortegaard LS, Hallas J, Moreno SI. The use of medication against attention deficit/ hyperactivity disorder in Denmark: a drug use study from a patient perspective. Eur J Clin Pharmacol. 2013;69(3):589-98.

25. Didoni A, Sequi M, Panei P, Bonati M. One-year prospective follow-up of pharmacological treatment in children with attention-deficit/hyperactivity disorder. Eur J Clin Pharmacol. 2011;67(10):1061-7.

26. Hosmer DW, Lemeshow S. Applied logistic regression. New York: Wiley; 2000.
27. Faber A, Kalverdijk LJ, de Jong-van den Berg LT, Hugtenburg JG, Minderaa RB, Tobi H. Co-morbidity and patterns of care in stimulant-treated children with ADHD in the Netherlands. Eur Child Adolesc Psychiatry. 2010;19(2):159-66.

28. Olfson M. New options in the pharmacological management of attention-deficit/hyperactivity disorder. Am J Manag Care. 2004;10(4 Suppl):S117-24.

29. Kratochvil C. New ADHD treatment options on the horizon. Adv Stud Med. 2002;2(25):915-8.

30. Kovshoff $\mathrm{H}$, Williams $\mathrm{S}$, Vrijens $\mathrm{M}$, et al. The Decisions Regarding ADHD Management (DRAMa) study: uncertainties and complexities in assessment, diagnosis and treatment, from the clinician's point of view. Eur Child Adolesc Psychiatry. 2012; 21(2):87-99. 